\title{
Mechanical Design Studies of the MQXF Long Model Quadrupole for the HiLumi LHC
}

\author{
H. Pan, E. Anderssen, G. Ambrosio, D. W. Cheng, M. Juchno, P. Ferracin, H. Felice, J. C. Perez, S. O. Prestemon, \\ G. Vallone
}

\begin{abstract}
The Large Hadron Collider Luminosity upgrade (HiLumi) program requires new low- $\beta$ triplet quadrupole magnets, called MQXF, in the Interaction Region (IR) to increase the LHC peak and integrated luminosity. The MQXF magnets, designed and fabricated in collaboration between CERN and the U.S. LARP, will all have the same cross section. The MQXF long model, referred as MQXFA, is a quadrupole using the Nb3Sn superconducting technology with $150 \mathrm{~mm}$ aperture and a $4.2 \mathrm{~m}$ magnetic length and is the first long prototype of the final MQXF design. The MQXFA magnet is based on the previous LARP HQ and MQXFS designs. In this paper we present the baseline design of the MQXFA structure with detailed 3D numerical analysis. A detailed tolerance analysis of the baseline case has been performed by using a 3D finite element model, which allows fast computation of structures modelled with actual tolerances. Tolerance sensitivity of each component is discussed to verify the actual tolerances to be achieved by vendors. Tolerance stack-up analysis is presented in the end of this paper.
\end{abstract}

Index Terms-High Luminosity LHC (HL-LHC), quadrupole, LARP, $\mathrm{Nb}_{3} \mathrm{Sn}$ magnet, shell-based support structure, long model, tolerance analysis.

\section{INTRODUCTION}

$\mathrm{H}$ IGH field large aperture quadrupoles are the key components required in the Interaction Region (IR) for the HiLumi LHC upgrade[1]. The new $150 \mathrm{~mm}$ aperture $\mathrm{Nb}_{3} \mathrm{Sn}$ low- $\beta$ quadrupole magnets, called MQXF are being developed by collaboration between the CERN HL-LHC project and the US-LARP (LHC Accelerator Research Program). The MQXF quadrupoles feature an aperture of 150 $\mathrm{mm}$ and provide a nominal field gradient of $132.6 \mathrm{~T} / \mathrm{m}$ by utilizing $\mathrm{Nb}_{3} \mathrm{Sn}$ superconductor over a magnetic length of 4.2 $\mathrm{m}$ (MQXFA) and $7.15 \mathrm{~m}$ (MQXFB) at cold [2].

In the framework, the US is in charge of the ten Q1 and Q3 cold masses [2]. To successfully start the long MQXF production, the HiLumi-LHC collaboration adopts a two-step process with the fabrication, assembly and test of the short (MQXFS) and the long prototypes (MQXFA) to systematically reduce the risk [3]. The short and long models of MQXF all have the same cross section.

Two short models (MQXFS1 and MQXFS3) have been

Manuscript received September 5, 2016. This work was supported by the, U.S. Department of Energy, Office of Science under contract No. DE-AC0205CH11231 and under Cooperative Agreement DE-SC0000661.

H.Pan (email:hengpan@lbl.gov), E. Anderssen, D. W. Cheng, M. Juchno, S. O. Prestemon, are with the Lawrence Berkeley National Laboratory, Berkeley, CA 94720 USA.

P. Ferracin, J.C. Perez, and G. Vallone are with CERN, 1211 Geneva, Switzerland.

G. Ambrosio is with FNAL, Batavia, IL 60510 USA

H. Felice is with CEA, 91190 Saclay, France

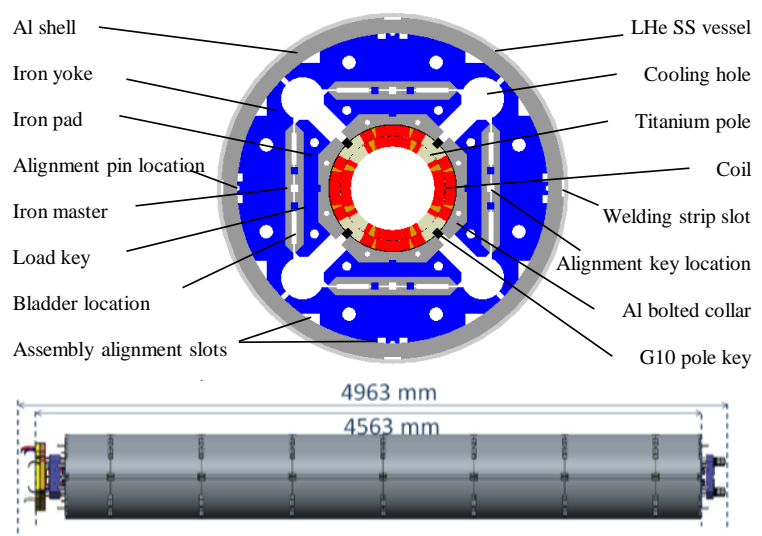

Fig. 1 Cross section and total length of MQXFA magnet

assembled and tested at $1.9 \mathrm{~K}$ recently [4]; MQXFS1 test results show a good agreement between strain measurement and FE model predictions [5], [6]. The long model adopts the major structure of MQXFS; we present in this paper the main parameters of the long model, with an analysis of the mechanical behavior from assembly to excitation. The mechanical tolerance analysis of the support structure is then described, pointing out the potential tolerance relaxation related to fabrication and assembly.

\section{MEChANICAL DESIGN OF MQXF}

In order to counteract nominal forces of $+2.47 /-3.48 \mathrm{MN} / \mathrm{m}$ $\left(\mathrm{F}_{\mathrm{x}} / \mathrm{F}_{\mathrm{y}}\right)$ without risking any overstress during assembly in the brittle Nb3Sn coils, the design for MQXF long model uses a shell-based support structure with the "bladder and key" concept, which is developed at LBNL for strain sensitive material such as $\mathrm{Nb}_{3} \mathrm{Sn}$. The performance of this support structure has been demonstrated by LARP HQ, LQ and MQXFS1 magnet [3][4][7]. The MQXFA support structure remains the same cross-section of MQXFS1 magnets, and scales-up in magnetic length. The magnet schematic of MQXFA in Fig. 1 shows same configurations in cross-section, but the actual length is extended to 4.2 magnetic length. Table I lists the major parameters of MQXF magnet.

As applied in the LARP HQ and MQXFS magnets, radial preloading in MQXFA relies on a system of water-pressurized bladders and keys to apply a partial pre-load to coil-pack and pre-tension to aluminum shell at room temperature. During the preload operation, the pressurized bladders open up the master pack and allow inserting the load keys with shims of the designed interference. The final pre-load is achieved during the cool-down phase, when the tensioned aluminum shell compresses the structure components because of its high thermal contraction. 
TABLE I

MQXF COIL AND MAGNET PARAMETERS

\begin{tabular}{lcc}
\hline \hline Parameter & Units & \\
\hline Structure length with splice box & $\mathrm{mm}$ & 4963 \\
Magnet (LHe vessel) outer diameter & $\mathrm{mm}$ & 630 \\
Coil clear Aperture diameter & $\mathrm{mm}$ & 150 \\
No. turns in layer1/2 (octant) & $\mathrm{T} / \mathrm{m}$ & $22 / 28$ \\
Nominal gradient $G_{\text {nom }}$ & $\mathrm{kA}$ & 132.6 \\
Nominal current $I_{\text {nom }}$ & $\mathrm{T}$ & 16.47 \\
Nominal conductor peak field $B_{\text {nom }}$ & $\mathrm{MJ} / \mathrm{m}$ & 1.4 \\
Stored energy density in straight sect. at $I_{\text {nom }}$ & $\mathrm{mH} / \mathrm{m}$ & 8.21 \\
Differential inductance at $I_{\text {nom }}$ & $\mathrm{MN} / \mathrm{m}$ & $+2.47 /-3.48$ \\
$\mathrm{~F}_{\mathrm{x}} / \mathrm{F}_{\mathrm{y}}$ (per octant) at $I_{\text {nom }}$ & $\mathrm{MN}$ & 1.17 \\
$\mathrm{~F}_{\mathrm{z}}$ (entire magnet) at $I_{\text {nom }}$ & & \\
\hline \hline
\end{tabular}

In the axial direction (parallel to the magnet's bore), prestress is also designed to withstand the total Lorentz forces generated by the coil ends on the order of $1.17 \mathrm{MN}$ at nominal current. In the present design of MQXFA, four tensioned steel rods within the yoke's cooling holes are connected to endplates. As the radial pre-stress, the initial axial pre-stress is tuned as to counteract $0.55 \mathrm{MN}$ after cool down.

\section{NUMERICAL ANALYSIS}

\section{A. Baseline case}

A fully parametric FE model of MQXFA was developed in ANSYS on basis of the previous MQXFS model [8]. Elements were generated using a volume sweep of 20-node structural element (SOLID186). The contact areas between the assembly components were modeled with TARGE170 and CONTA174 elements with asymmetric behavior and augmented Lagrange formulation [9]. Friction coefficient of 0.2 was used at the interfaces of support components. Material properties are listed in Table II. The entire operation process was simulated by the following four steps:

1) Key shimming: the interference shim was applied as the contact offset between load key and master pad.

2) Cool-down to $1.9 \mathrm{~K}$ : the temperature of all solids was changed from $300 \mathrm{~K}$ to $1.9 \mathrm{~K}$.

3) Magnetic excitation to nominal gradient $130 \mathrm{~T} / \mathrm{m}$ (rounded from the value of Table I): Import the coil from ROXIE to opera, and then compute forces in opera and import from opera to ANSYS.

4) Magnetic excitation to maximum gradient $140 \mathrm{~T} / \mathrm{m}$ : scale the imported Lorentz force to the level of the maximum gradient.

TABLE II

MQXF MATERIAL PROPERTIES

\begin{tabular}{llll}
\hline \hline \multirow{2}{*}{ Material } & $\mathrm{E}[\mathrm{GPa}]$ & $\begin{array}{l}\text { Poisson } \\
\text { ratio }\end{array}$ & $\alpha(293 \mathrm{~K}->4.3 \mathrm{~K})$ \\
\cline { 2 - 3 } & $293 \mathrm{~K} / 4.2 \mathrm{~K}$ & 0.3 & $3.36 \mathrm{e}-3$ \\
Coil & $20 / 20$ & 0.28 & $2.84 \mathrm{e}-3$ \\
Stainless steel & $193 / 210$ & 0.28 & $1.97 \mathrm{e}-3$ \\
Iron & $213 / 224$ & 0.34 & $4.2 \mathrm{e}-3$ \\
Aluminum & $70 / 79$ & 0.3 & $7.06 \mathrm{e}-3$ \\
G10 (Wrap) & $17 / 17$ & 0.3 & $1.74 \mathrm{e}-3$ \\
Titanium & $130 / 130$ & 0.28 & $2.6 \mathrm{e}-3$ \\
Nitronic 50 & $210 / 225$ & 0.3 & $3.12 \mathrm{e}-3$ \\
Al Bronze & $110 / 120$ & & \\
\hline \hline
\end{tabular}

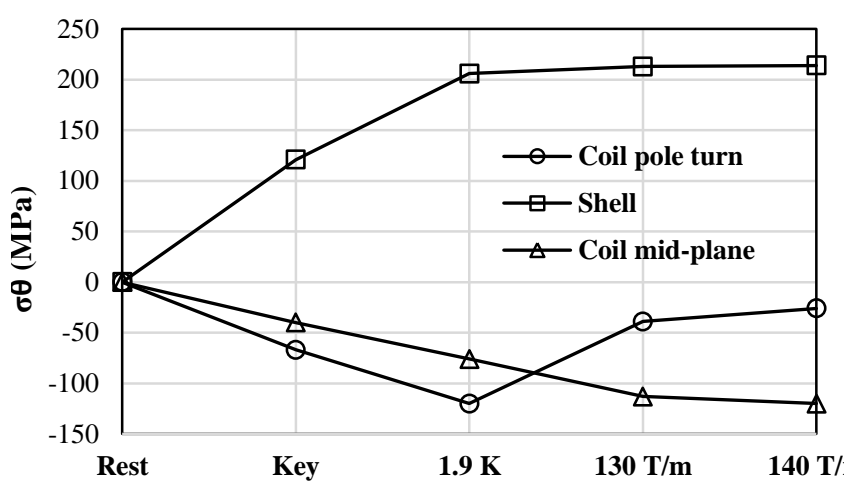

Fig. 2 Azimuthal stress in the coil inner layer from assembly to excitation: mid-radius of the pole turn (round markers), mid-radius of the mid-plane turn (triangle markers), and the shell stress (square markers).

Ensuring proper preload of the coils is paramount for the magnet performance. An interference of $850 \mu \mathrm{m}$ was chosen as the baseline case to preserve the nominal coil shape allowing excessive stress at the maximum gradient of 140 $\mathrm{T} / \mathrm{m}$. Axial preload in the model is provided by pre-tensioning $-516 \mu \varepsilon$ on the axial steel rods.

The average azimuthal stress evolutions in the coil inner layers and shell are given in Fig. 2. The round, triangle, and square markers indicate the azimuthal stresses, respectively at the mid-radius of the pole turn (inner layer, center of the turn), at the mid-radius of the mid-plane turn and at $15^{\circ}$ from the welding strip slot of the shells. During assembly, pre-stressing was stopped with $120 \mathrm{MPa}$ of tension in the shell. It increased to $208 \mathrm{MPa}$ during cool-down. The shell's tension is azimuthally uniform, except for some curvature changes near the iron gaps. The inner coil's azimuthal stress reaches -60 $\mathrm{MPa}$ (compression) during assembly, increasing to $-125 \mathrm{MPa}$ with cool-down. With Lorentz force, the stress in the shell is nearly constant, while the stress in the coil varies linearly with the square of the current.

In terms of peak stress, the coil reaches a maximum compression of $-129 \mathrm{MPa}$ during room temperature bladder operation, $-192 \mathrm{MPa}$ in the pole region at $1.9 \mathrm{~K}$ which remains safely below $200 \mathrm{MPa}$ criteria [10], and -165 MPa on the mid-plane with Lorentz forces at $140 \mathrm{~T} / \mathrm{m}$.

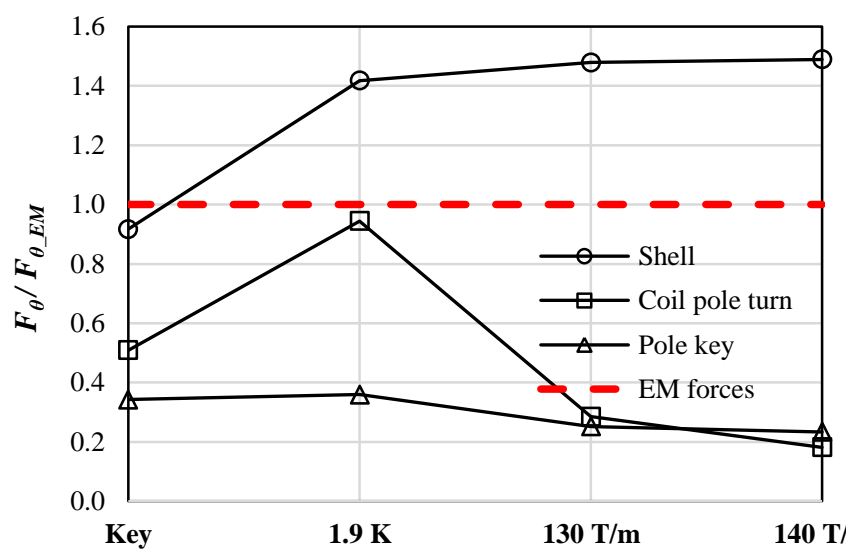

Fig. 3 Ratio between azimuthal total e.m. force per octant [2] and the azimuthal force provided by the shell and received by the coil and pole key. 


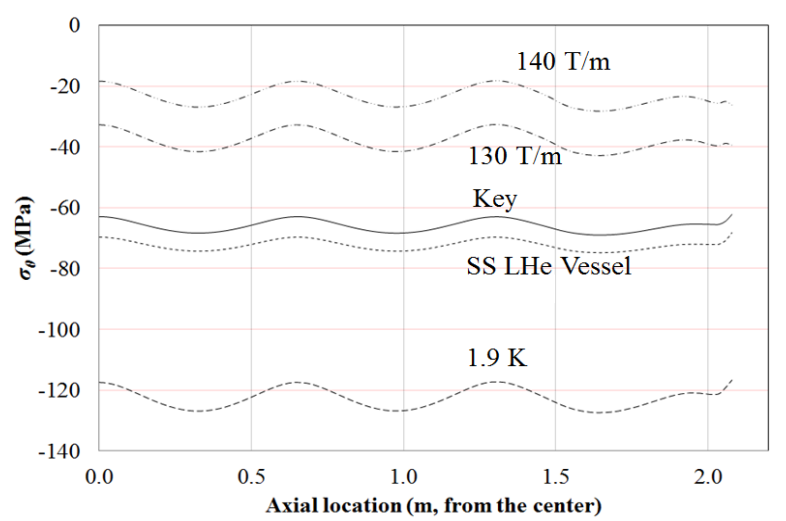

Fig. 4 Coil azimuthal stress of mid-radius of the pole turn (inner layer)

At $1.9 \mathrm{~K}$, all the iron parts exhibit first principal stresses below $300 \mathrm{MPa}$ with a safety factor of 2.41 . The shell azimuthal stress remains below $350 \mathrm{MPa}$ after cool-down. From the standpoint of coil stress, the baseline case reveals that the $850 \mu \mathrm{m}$ interference could be the upper limit of the practical radial shim.

Fig. 3 presents how the mechanical forces within the magnet evolve over the operation process. During the assembly, 55\% of the force provided by the shell is transferred to the coil, the rest being intercepted by the pole key. After cool-down, the shell loads the coil to the target value based on the e.m. force at the maximum gradient. In this design, the pole key intercepts the similar fraction of the shell force at 1.9 $\mathrm{K}$ by orientating the key cloth fibers parallel to the azimuthal direction to prevent the pole key from losing due to higher thermal contraction in the normal direction. The coil pole turns are still under compression with the Lorentz forces; the compressive force on the pole key reduced a bit with Lorentz forces, but still indicates that coil alignment is maintained.

\section{B. Impact of the SS LHe vessel}

Because the segmented shell cannot provide LHe containment, an additional $8 \mathrm{~mm}$ thick stainless steel shell will be installed outside the support structure [11]. In order to ensure it to maintain the contact with the structure after cooldown, additional pre-tension has to be applied accounting for the lower thermal contraction compared with the aluminum shells. Consequently, this additional SS vessel also provides additional pre-load to the structure.

With the updated 3D model, the steel vessel was pretensioned to $100 \mathrm{MPa}$. Fig. 4 indicates the pre-tensioned $\mathrm{LHe}$ vessel slightly improved the coil stress uniformity along the magnet length. The coil stress variation is about $\pm 4.25 \mathrm{MPa}$ (9.5 MPa peak-to-peak) after cool-down with the SS LHe vessel. The increase of stress in the coil is of the order of 5.5 MPa at $140 \mathrm{~T} / \mathrm{m}$, which could be taken into account during assembly.

\section{Mechanical Tolerance ANALysis}

During the assembly, the pressurized bladders compress the coil-pack and allow shimming the load keys, placed between the iron yoke and the iron pad, with interference shims. For the practical assembly with tolerances in each part (the tolerance in this study refers to the linear bilateral profile tolerance only), we evaluated the impact of the tolerance stack-up in radial directions on the coil stress deviation with the given interference.

Tolerance analysis in this study is based on the linear dimensional chain calculation. A dimensional chain is a set of independent parallel dimensions which continue each other to create a geometrically closed circuit. In the case of MQXFA magnet, the radial dimensional chain consists of the radial dimensions of the collar, the pad, the masters, the yoke and the shell, called input dimensions and the gap between the shimming key and the pad-master, called the resulting dimension. Fig. 5 shows how the radial dimensional chain builds up in MQXFA, $L x$ is the resulting dimension in the dimension loop.

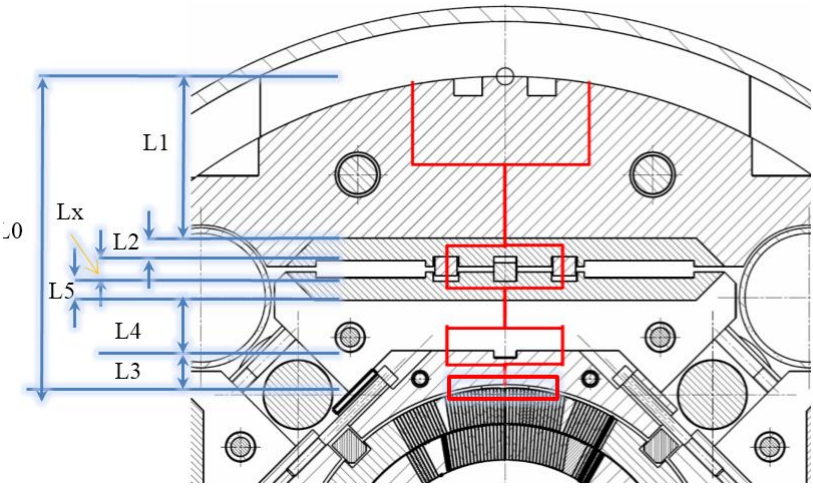

Fig. 5 Schematic of radial tolerance chain; L1 to L5 represent the radial dimensions of the yoke, yoke-master plate, collar, pad and padmaster plate;

\section{A. $\quad$ Tolerance Sensitivity Study}

Each of the parts that stacked on top of the coils will affect the coil stress, and all of the tolerances associated on those parts need to be managed. When it comes to manufacturing, the requirement of each part is certainly different considering the part's function and cost. While assembling the magnet, it also asks for which tolerance has the most impact on the coil stress.

The logic of the tolerance sensitivity study is to find the coil stress deviation with individual tolerance of $25 \mu \mathrm{m}$ (which is close to the manufacturing tolerances), and then find out which tolerance results the maximum stress deviation. A full size 2D ANSYS model was created to simulate the mechanical

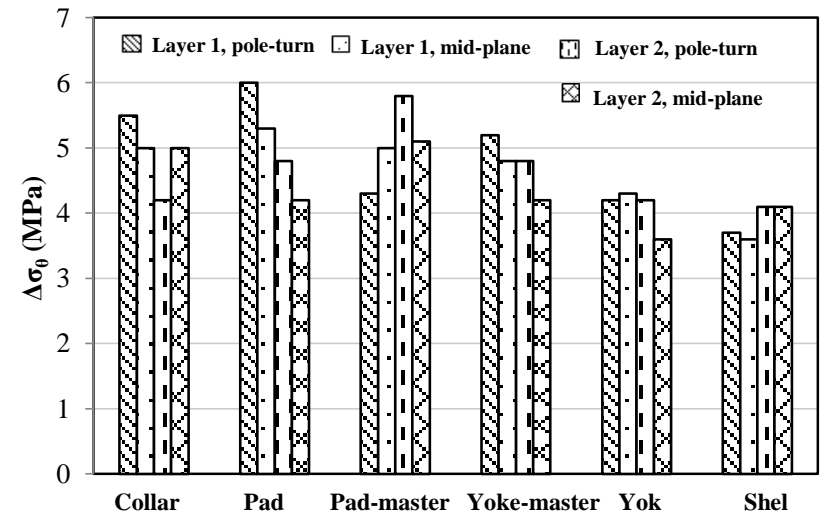




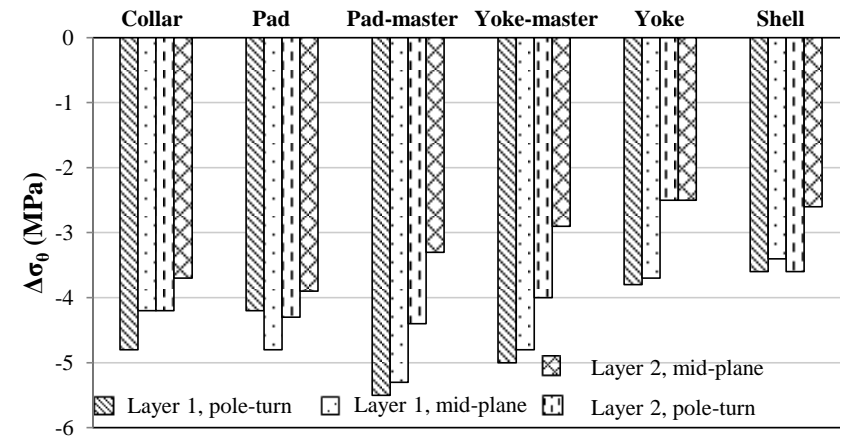

Fig. 6 (a), (b) Coil azimuthal stress deviation of $+/-25 \mu \mathrm{m}$ tolerance on each individual part

behavior with applying tolerances. In this study, there are no symmetry boundary conditions inside the magnet; therefore, it allows applying different tolerances on selected quadrants.

In this study, the objective parameters are the average coil azimuthal stress in the following locations: (a) layer 1, coil pole turn $\left(\sigma_{I p}\right)$; (b) layer 1, coil mid-plane $\left(\sigma_{I m}\right)$; (c) layer 2, coil pole turn $\left(\sigma_{2 p}\right) ;\left(\right.$ d) layer 2 , coil mid-plane $\left(\sigma_{2 m}\right)$. Fig. 6 presents how sensitive of each structure component to their own profile tolerance in both directions. For each given tolerance of $\pm 25 \mu \mathrm{m}$ in individual part, the coil average stresses respond within $\pm 6 \mathrm{MPa}$. The tolerances of the padmaster and pad have a relatively larger impact on the coil stresses; Yoke and shell are less sensitive to the tolerance, which indicates that the tolerance on those parts could be released compared to the pad and masters.

The maximum stress in the structure components spears in excitation. In the analysis, the coils are assumed as in nominal size, which implies the analysis ignores the coil shift due to the broken symmetry. Further calculation will involve detailed coil model with cables to calculate the field with shifted coil.

TABLE III

MAX COIL STRESS DEVIATION

\begin{tabular}{ccccccc}
\hline \hline & Collar & Pad & $\begin{array}{c}\text { Pad- } \\
\text { master }\end{array}$ & $\begin{array}{c}\text { Yoke- } \\
\text { master }\end{array}$ & Yoke & Shell \\
\hline $\begin{array}{c}\Delta \sigma_{\max } \\
(\mathrm{MPa})\end{array}$ & 4 & 4.6 & 4.9 & 4.2 & 3.3 & 3.1 \\
\hline \hline
\end{tabular}

In terms of the influence on the coil peak stress, padmaster is also the most sensitive part. Table III lists the maximum coil stress deviation with each individual tolerance. It is apparent that the pad master and pad exhibit the most influence on coil stress.

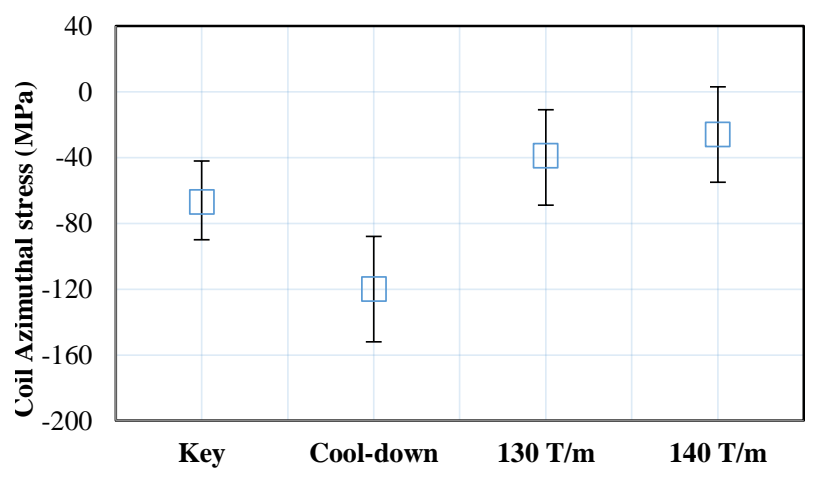

Fig. 7 Coil azimuthal stress with the deviation in the WC (Worst Case) tolerance stacking

\section{B. $\quad$ Coil stress with radial tolerance build-up}

To analyze tolerance stack-up in an assembly, tolerance stacking method has to be defined ahead of analysis. There are two well-known ways to stack tolerances: Worst Case (WC) tolerance stacking and Statistical tolerance.

The Worst case method, also known as linear stack-up, is the most basic method for predicting the effect of individual tolerances on the whole assembly [12].

$$
\Delta T=\sum_{k=1}^{n} T_{k}
$$

Where, $n=$ Number of constituent dimensions in the dimension chain, $T_{k}=$ Tolerance associated with dimension.

From expression (1), the overall tolerance stack ups is computed as $\pm 275 \mu \mathrm{m}$ if each individual tolerance is $25 \mu \mathrm{m}$. Fig. 7 shows the deviation band of the azimuthal stress at the mid-radius of the coil pole turn of the $1^{\text {st }}$ layer. The maximum bandwidth in one direction is about $32 \mathrm{MPa}$. The weakness of the method is that its predictions become too conservative, because as the number of the parts in the assembly increases then the chances of all the individual tolerances occurring at their worst case limits reduce.

Statistical tolerancing, also refers to RSS (Root Sum Squares), assumes a probability distribution function for the variation of tolerances and then uses this function to predict the assembly variability in the system. This case is frequently used in mechanical assemblies because it is close to manufacturing experiences [13]. Total tolerance of assembly can be given as

$$
\Delta T=\sqrt{\sum_{k=1}^{n} T_{k}^{2}}
$$

The computed radial tolerance stack up is $83 \mu \mathrm{m}$ by the RSS method, which is considerably lower than the WC case. The resultant maximum deviation bandwidth of the coil stress is about $9 \mathrm{MPa}$ in one direction.

\section{CONCLUSION}

The mechanical analysis of MQXFA magnet has been analyzed in 3D, from assembly, through cool-down and magnetic loading. The support structure of MQXFA adopts the same design concept demonstrated in MQXFS1 prototypes. The baseline interference for the case of the maximum gradient operation is set $850 \mu \mathrm{m}$. The mechanical impact of stainless steel shell is checked as well in this study. The mechanical tolerances of each individual part affect the coil stress slightly. Pad and pad master are the most sensitive parts among the structure components. The maximum coil stress deviation is $\pm 32 \mathrm{MPa}$ at cold in the worst case; however, based on the manufacturing experiences, the coil stress deviation could be lowered to \pm 9 MPa with RSS method.

\section{ACKNOWLEDGEMENT}

Work supported by the US Department of Energy through the US LHC Accelerator Research Program (LARP) and by the High Luminosity LHC Project at CERN. 


\section{REFERENCES}

[1] G. Ambrosio, "Nb3Sn High Field Magnets for the High Luminosity LHC Upgrade Project," IEEE Trans. Appl. Supercond., vol. 25, pp.4002107, June 2015.

[2] P. Ferracin, et al, "Development of MQXF: The Nb3Sn Low- $\beta$ Quadrupole for the HiLumi LHC," IEEE Trans. Appl. Supercond., vol. 26, pp.4000207, June 2016.

[3] H. Pan et al., "Assembly tests of the first $\mathrm{Nb}_{3} \mathrm{Sn}$ low-beta quadrupole short model for the Hi-Lumi LHC upgrade," IEEE Trans. Appl. Supercond., vol. 26, pp. 4001705, Jun. 2016.

[4] G. Vallone, et al, "Mechanical Performance of Short Models for MQXF, the Nb3Sn Low- $\beta$ Quadrupole for the Hi-Lumi LHC," to be published.

[5] G. Vallone, et al, "MQXFS1 strain gauge analysis," Report. Joint LARP CM26 / Hi-Lumi Meeting, SLAC. May, 2016.

[6] G. Vallone, et al, "Mechanical Performance of Short Models for MQXF, the Nb3Sn Low- $\beta$ Quadrupole for the Hi-Lumi LHC," IEEE Trans. Appl. Supercond., submitted, Oct. 2016.

[7] S. Caspi et al., "Design of a $120 \mathrm{~mm}$ bore $15 \mathrm{~T}$ quadrupole for the LHC upgrade phase II," IEEE Trans. Appl. Supercond., vol. 20, no. 3, pp. 144-147, Jun. 2010.

[8] H. Pan. "Overview of FE models and updates of the MQXF prototype structures," report. MQXF Workshop on Structure, Alignment, and Electrical QA, CERN, Feb. 2016

[9] ANSYS, "ANSYS help document", release 2016, 2016

[10] H. Felice, et al, "Design of the MQXFA 1st prototype support structure", Report. LARP Internal Review of the MQXF Mechanical Structure Design and Functional Requirments, LBNL. July, 2015.

[11] M. Juchno, et al., "Support Structure Design of the Nb3Sn Quadrupole for the High Luminosity LHC," IEEE Trans. Appl. Supercond., vol. 25, no. 3, pp. 1-4, Jun. 2015.

[12] F. Scholz, "Tolerance Stack Analysis Methods," Research and Technology Boeing Information \& Support Services, Boeing, Seattle, pp 1-44, December, 1995

[13] D. H. Evans, "Statistical tolerancing: the state of art. Part II: Methods for estimating moments". Journal of Qual Technol, vol 7(1), pp:1-12, 1975 